Case Report

\title{
Neurological Manifestations of X-Linked Ichthyosis: Case Report and Review of the Literature
}

\author{
William S. Baek ${ }^{1}$ and Umut Aypar ${ }^{2}$ \\ ${ }^{1}$ Parkside Medical Group, 1310 San Bernardino Rd, Suite 102, Upland, CA 91786, USA \\ ${ }^{2}$ Division of Laboratory Genetics, Department of Laboratory Medicine \& Pathology, Mayo Clinic, 200 First Street SW, \\ Rochester, MN 55905, USA
}

Correspondence should be addressed to William S. Baek; william_s_baek@hotmail.com

Received 8 February 2017; Revised 2 May 2017; Accepted 12 July 2017; Published 13 August 2017

Academic Editor: Balraj Mittal

Copyright (C) 2017 William S. Baek and Umut Aypar. This is an open access article distributed under the Creative Commons Attribution License, which permits unrestricted use, distribution, and reproduction in any medium, provided the original work is properly cited.

A 5-year-old boy presented with mild autism and attention-deficit hyperactivity disorder (ADHD). Chromosomal microarray demonstrated a 1.7 Mb deletion at Xp22.31, which was consistent with X-linked ichthyosis (XLI). Further exam revealed dry, scaly skin on his abdomen and pretibial areas. Patients with mutations involving solely the STS gene or the recurrent $\sim 2 \mathrm{Mb}$ deletion may present with ADHD, whereas those with larger deletions including the NLGN4 gene can present with both ADHD and autism. However, our patient presented with mild autism in addition to ADHD despite having only the recurrent deletion without loss of NLGN4. Such neurological manifestations of XLI warrant attention as practical targets of clinical management.

\section{Introduction}

$\mathrm{X}$-linked ichthyosis (XLI) is an X-linked recessive disorder with a prevalence of approximately $1: 4,000$, demonstrating dry, scaly skin due to a deficiency of the enzyme steroid sulfatase [1]. While patients with mutations involving solely the STS gene or the recurrent $\sim 2 \mathrm{Mb}$ deletion can present with attention-deficit hyperactivity disorder (ADHD), those with larger deletions including neighboring genes such as neuroligin 4 (NLGN4) may present with autism in addition to $\mathrm{ADHD}[2,3]$.

We present a case of XLI with ADHD and mild autism due to a recurrent $\sim 2 \mathrm{Mb}$ deletion which spared the NLGN4 gene.

\section{Case Presentation}

A 5 -year-old boy ( 43 inches, 50th percentile, and $42 \mathrm{lbs}$, 50 th percentile) presented to Parkside Medical Group (Upland, CA) for a neurological evaluation. His mother remembered being told that he might have intellectual disability based on blood tests and an amniocentesis; however, these test results were not available. Otherwise, the pregnancy history and delivery were normal. He weighed $6 \mathrm{lbs} 10 \mathrm{oz}$ (20th percentile) at birth, walked at 14 months, and said "mama" and "papa" at 8-9 months. During early childhood he did not want to be around other children. He adhered to routines; for example, when his father would change his favorite TV channel he would become very angry. He spoke Spanish and some English. He attended special education at a preschool level and he could count up to 100. However, he could not focus in school. He was diagnosed with ADHD and mild autism based on the DSM V criteria by a child psychologist six months prior to the neurology visit. He was started on amphetamine-dextroamphetamine ER $10 \mathrm{mg}$ daily, which improved his behavior both at home and at school. He started participating more in group activities. On exam, he was found to have a high anterior hairline, a triangular face, a short chin, widely spaced eyes, prominent nasal bridge, and conical teeth (Figure 1). There were no corneal opacities or preauricular scales. He was able to answer questions about his name, age, and what he liked, but there was a lack of reciprocity and eye contact. There were no tics. However, he was fidgety, could not wait his turn, was wandering about, was talking 


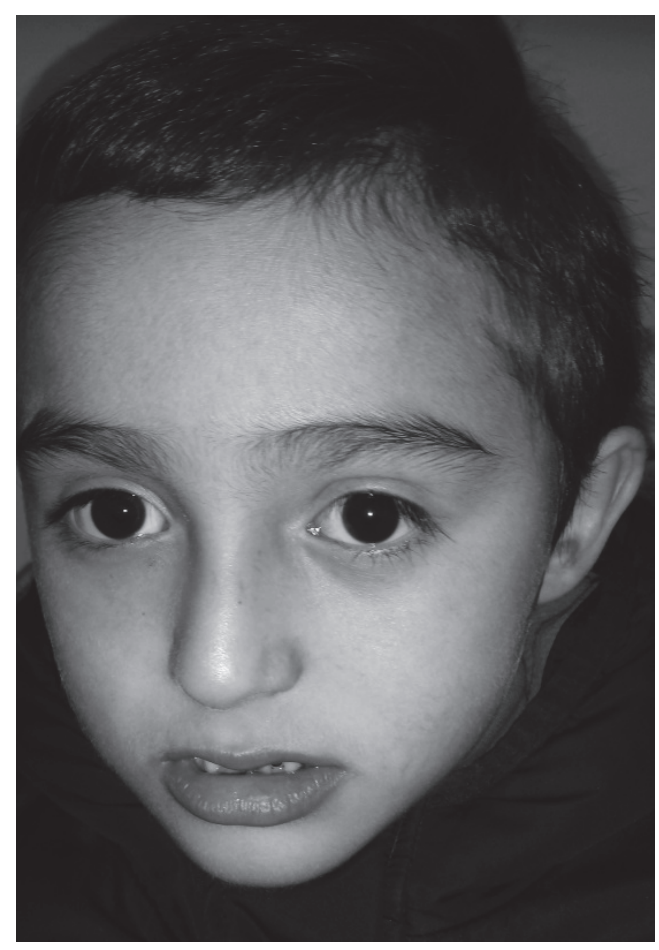

(a)

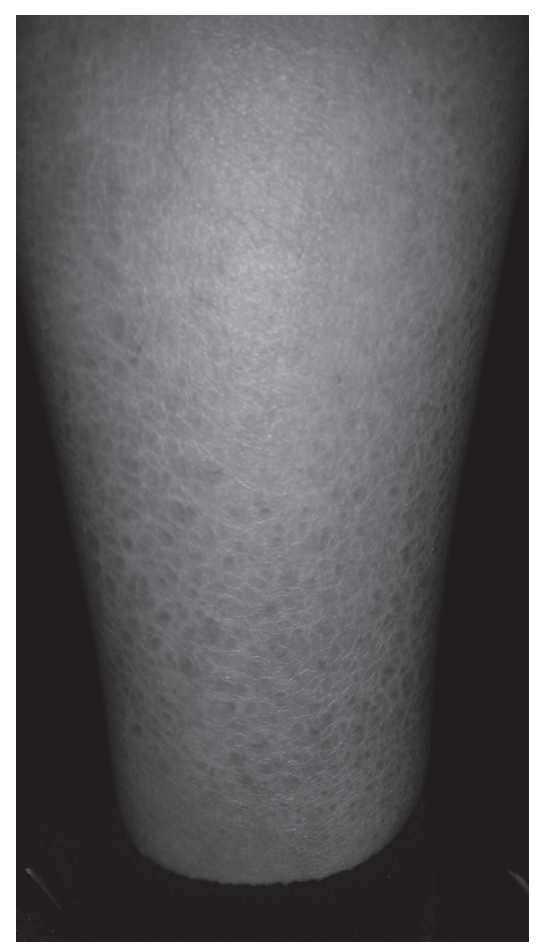

(b)

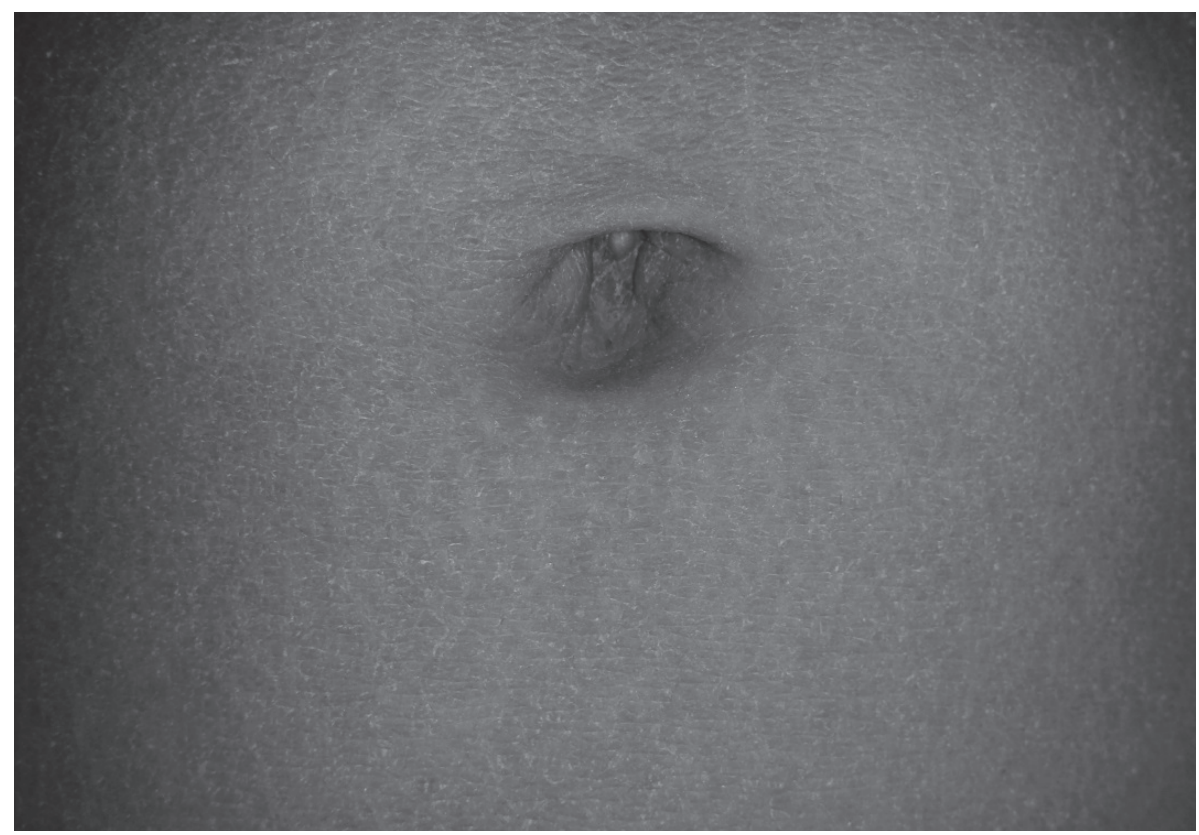

(c)

FIGURE 1: (a) Facial features include the high anterior hairline, a triangular face, a short chin, widely spaced eyes, and prominent nasal bridge. The dry, scaly skin was observed in (b) the pretibial areas and (c) the abdomen.

excessively in Spanish, and was interrupting his parents at times. Therefore, his Adderall XR was increased to $20 \mathrm{mg}$ daily, but he became weepy and emotional; hence the dose was lowered back to $10 \mathrm{mg}$ daily, but 7 months later the dose had to be increased back to $15 \mathrm{mg}$ daily due to his persistent symptoms of ADHD. He was also treated for insomnia with guanfacine $0.5 \mathrm{mg}$ at night. Of note, he had a history of asthma and was being treated with budesonide $0.5 \mathrm{mg} / 2 \mathrm{~mL}$ suspension and montelukast $4 \mathrm{mg}$ daily. Family history was unremarkable. He has an 11-year-old healthy sister. There was no history of consanguinity. His EKG was normal. There was no history of seizures; EEG was not performed due to lack of cooperation. His lab results were all normal (cholesterol, TG, HDL, LDL, FSH, LH, TSH, ACTH, vitamin A, free retinol, 


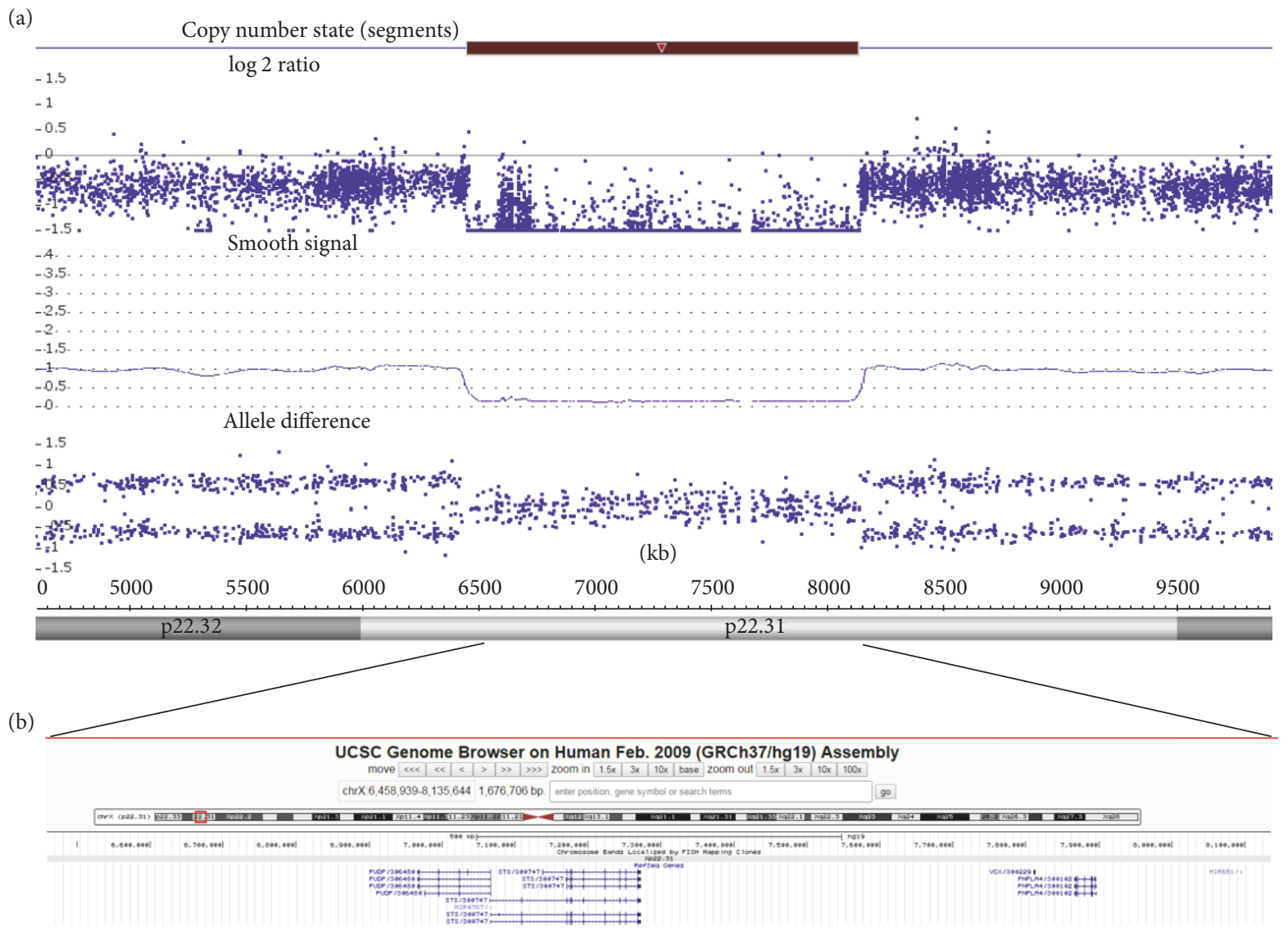

Figure 2: (a) Affymetrix CytoScan chromosomal microarray analysis detected a 1.7 Mb deletion at Xp22.31 (6,458,939-8,135,644, genome build hg 19) in our male patient. (b) UCSC genome browser image showing the Xp22.31 deletion. The deleted interval contains six known genes (PUDP, STS, MIR4767, VCX, PNPLA4, and MIR651).

and plac Lp PLA2) except for human growth hormone, which was elevated (1.6 ng/ml; normal range $0.01-0.97 \mathrm{ng} / \mathrm{ml})$. He tested negative for fragile X syndrome with 29 CGG repeats. Chromosomal microarray (CMA), performed at the Mayo Clinic (Rochester, MN), demonstrated a $1.7 \mathrm{Mb}$ deletion at Xp22.31 (Figure 2), which included 6 genes; STS, pseudouridine $5^{\prime}$-phosphatase (PUDP), microRNA 4767 (MIR4767), variable charge, X-linked (VCX), patatin-like phospholipase domain containing 4 (PNPLA4), and microRNA 651 (MIR651), which was consistent with the diagnosis of XLI [4, 5]. CMA was performed using both copy number and singlenucleotide polymorphism (SNP) probes on a whole genome array (CytoScan HD platform) (Affymetrix). The genomewide functional resolution of this array is approximately $30 \mathrm{~kb}$ for deletions and $60 \mathrm{~kb}$ for duplications. The presence of dry, scaly skin on his abdomen and pretibial areas (Figure 1) was confirmed after receiving the results of the array. There were no signs of cryptorchidism. The patient's mother could not be tested due to lack of insurance coverage.

\section{Discussion}

Here we present a case of XLI with 1.7 Mb deletion at Xp22.31 which included the STS gene, as well as 5 other genes. In addition to the classic features of XLI of dry, scaly skin (Figure 1), he also presented with ADHD and mild autism. During the pregnancy, his mother recalls some blood tests that are not available but presumably they showed low or undetectable serum levels of unconjugated estriol, which can be seen in XLI.

XLI was first described by Sedgwick in 1863 [6]. XLI is $\mathrm{X}$-linked recessive; therefore, it occurs almost exclusively in boys with a prevalence of approximately $1: 4,000$ [1]. It is due to a lack of the enzyme steroid sulfatase as a result of mutations or deletions of the STS gene, which is located in the distal part of the short arm of the X chromosome (Xp22.31) [7]. XLI presents with dry, scaly skin (ichthyosis), skin lesions, corneal opacities, and cryptorchidism [1]. Patients with mutations involving only the STS gene or the recurrent $\sim 2 \mathrm{Mb}$ deletion may present with ADHD, whereas those with larger deletions (involving not only STS or genes in the recurrent $\sim 2 \mathrm{Mb}$ deletion but also other neighboring genes such as NLGN4) may present with ADHD and/or autism $[2,3]$. Since patients with only STS mutations or recurrent $\sim$ $2 \mathrm{Mb}$ deletions have not demonstrated any autistic features, it was considered that the loss of one or more of the neighboring genes within the larger deletions is contributing to autism [3]. 
In our patient, chromosomal microarray revealed the recurrent $\sim 2 \mathrm{Mb}$ deletion, which included the following genes: STS, PUDP, MIR4767, VCX, PNPLA4, and MIR651. The significance of PUDP, MIR4767, and MIR651 is uncertain as there is not much known about the biological role of these genes. PUDP encodes a member of the haloacid dehalogenase-like (HAD) hydrolase superfamily; however, the encoded protein has no known biological function [8]. There is no information available on MIR4767 and MIR651 with regard to their targets [8]. VCX (variable charge, Xlinked) gene belongs to the VCX/Y gene family, all of which are expressed exclusively in male germ cells. While it is considered that the VCX/Y genes encode for small and highly charged nuclear proteins, their function is yet to be elucidated [8]. Although there have been conflicting reports on the association between VCX3A and an abnormal neurocognitive phenotype, no such correlation exists with the gene deleted in our patient (VCX) or with other gene family members (VCX2, VCX3B) [9, 10]. PNPLA4 encodes a member of the patatin-like family of phospholipases, which has both triacylglycerol lipase and transacylase activities. PNPLA4 may be involved in adipocyte triglyceride homeostasis [11]. It has also been implicated as a causal gene for autism [7]. STS belongs to the sulfatase family and hydrolyzes several 3-beta-hydroxysteroid sulfates, converting specifically the sulfated form of dehydroepiandrosterone, otherwise known as DHEA-S to DHEA [8]. These are both neurosteroids which exhibit effects on neurophysiological and behavioral processes. Lower levels of DHEA in the blood have been associated with ADHD phenotype [12]. This could explain the link between loss of STS gene function and ADHD in XLI patients. In addition, animal studies suggest that the STS gene may be implicated in processes involving attention [13].

Kent et al. [2] reported that, out of 25 male patients with either STS point mutations or recurrent $\sim 2 \mathrm{Mb}$ deletions, $40 \%$ fulfilled DSM-IV criteria for ADHD (compared to population rate; $3.6 \%$ ), $80 \%$ of which were inattentive subtype. Five had fulfilled criteria for an autistic spectrum disorder or related language/communication disorders, and all of them had unusually large deletions of the STS gene with loss of the NLGN4 gene. Males with solely STS mutations or recurrent $\sim 2 \mathrm{Mb}$ deletions did not demonstrate autistic difficulties. Therefore, NLGN4 was suggested as a causative gene for autism in XLI patients with larger deletions.

In our patient, however, the recurrent $\sim 2 \mathrm{Mb}$ deletion (including STS, PUDP, MIR4767, VCX, PNPLA4, and MIR651 but sparing NLGN4) was sufficient to cause both mild autism and ADHD.

Our case report highlights the importance of a neurological evaluation in XLI due to the association with $\mathrm{ADHD}$ and autism, especially those with the recurrent $\sim 2 \mathrm{Mb}$ deletions and those with larger deletions including NLGN4. In addition, we suggest that clinical cytogenetics laboratories which report these deletions should indicate the risk for the neurological manifestations in their interpretation, in addition to the skin manifestations and other clinical features. In conclusion, we hope to bring awareness of the neurological manifestations of XLI among neurologists and geneticists (both laboratory and clinical).

\section{Materials and Methods}

Genomic DNA extraction and chromosomal microarray analysis were performed with the previously described methods [14]. Informed consent was obtained from the mother for the photographs.

\section{Disclosure}

No statistical analysis was required.

\section{Conflicts of Interest}

The authors declare that they have no conflicts of interest.

\section{References}

[1] H. Traupe, J. Fischer, and V. Oji, "Nonsyndromic types of ichthyoses-an update," Journal of the German Society of Dermatology, vol. 12, no. 2, pp. 109-121, 2014.

[2] L. Kent, J. Emerton, V. Bhadravathi et al., "X-linked ichthyosis (steroid sulfatase deficiency) is associated with increased risk of attention deficit hyperactivity disorder, autism and social communication deficits," Journal of Medical Genetics, vol. 45, no. 8, pp. 519-524, 2008.

[3] K. J. Brookes, Z. Hawi, A. Kirley, E. Barry, M. Gill, and L. Kent, "Association of the steroid sulfatase (STS) gene with attention deficit hyperactivity disorder," American Journal of Medical Genetics, Part B: Neuropsychiatric Genetics, vol. 147, no. 8, pp. 1531-1535, 2008.

[4] A. Ballabio, M. Zollo, R. Carrozzo et al., "Deletion of the distal short arm of the X chromosome (Xp) in a patient with short stature, chondrodysplasia punctata, and X-linked ichthyosis due to steroid sulfatase deficiency," American Journal of Medical Genetics, vol. 41, no. 2, pp. 184-187, 1991.

[5] R. E. Schnur, B. J. Trask, G. Van den Engh et al., "An Xp22 microdeletion associated with ocular albinism and ichthyosis: Approximation of breakpoints and estimation of deletion size by using cloned DNA probes and flow cytometry," American Journal of Human Genetics, vol. 45, no. 5, pp. 706-720, 1989.

[6] W. Sedgwick, "On the Influence of Sex in Hereditary Disease," The British Journal of Psychiatry, vol. 9, no. 47, pp. 405-410, 1863.

[7] M. Carmen Carrascosa-Romero, J. Suela, B. Alfaro-Ponce, and A. J. Cepillo-Boluda, "X-chromosome-linked ichthyosis associated to epilepsy, hyperactivity, autism and mental retardation, due to the Xp22.31 microdeletion," Revista de Neurologia, vol. 54, no. 4, pp. 241-248, 2012.

[8] https://www.ncbi.nlm.nih.gov/refseq/.

[9] H. Ben Khelifa, N. Soyah, I. Ben-Abdallah-Bouhjar et al., "Xp22.3 interstitial deletion: A recognizable chromosomal abnormality encompassing VCX3A and STS genes in a patient with X-linked ichthyosis and mental retardation," Gene, vol. 527, no. 2, pp. 578-583, 2013.

[10] S. A. Cuevas-Covarrubias and L. M. González-Huerta, "Analysis of the VCX3A, VCX2 and VCX3B genes shows that VCX3A gene deletion is not sufficient to result in mental retardation in X-linked ichthyosis," British Journal of Dermatology, vol. 158, no. 3, pp. 483-486, 2008.

[11] P. A. Wilson, S. D. Gardner, N. M. Lambie, S. A. Commans, and D. J. Crowther, "Characterization of the human patatin-like 
phospholipase family," Journal of Lipid Research, vol. 47, no. 9, pp. 1940-1949, 2006.

[12] R. D. Strous, B. Spivak, R. Yoran-Hegesh et al., "Analysis of neurosteroid levels in attention deficit hyperactivity disorder," International Journal of Neuropsychopharmacology, vol. 4, no. 3, pp. 259-264, 2001.

[13] K. J. Brookes, Z. Hawi, J. Park, S. Scott, M. Gill, and L. Kent, "Polymorphisms of the steroid sulfatase (STS) gene are associated with attention deficit hyperactivity disorder and influence brain tissue mRNA expression," American Journal of Medical Genetics, Part B: Neuropsychiatric Genetics, vol. 153, no. 8, pp. 1417-1424, 2010

[14] Y. Cao and U. Aypar, "A novel Xq22.1 deletion in a male with multiple congenital abnormalities and respiratory failure," European Journal of Medical Genetics, vol. 59, no. 5, pp. 274-277, 2016. 


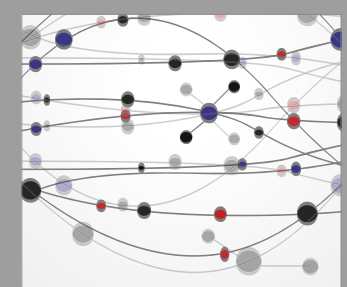

The Scientific World Journal
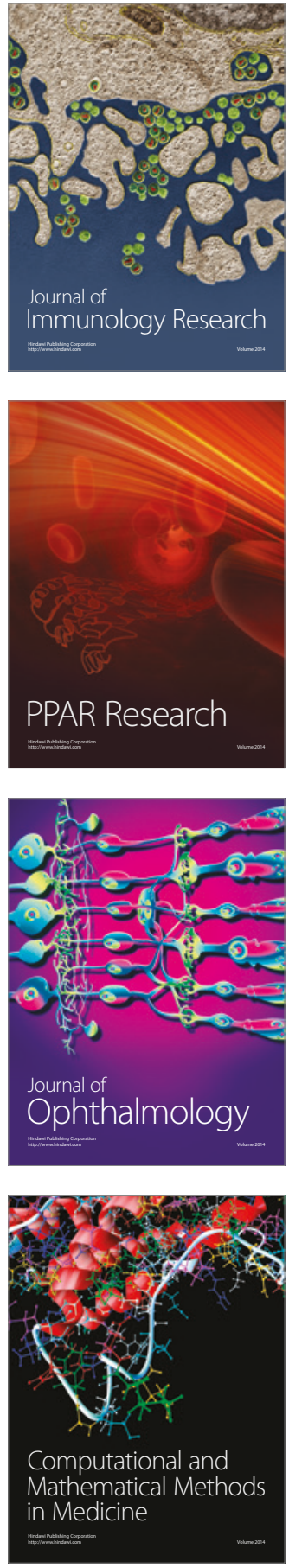

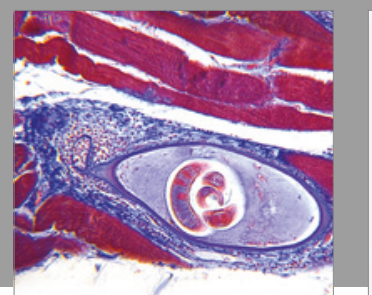

Gastroenterology Research and Practice
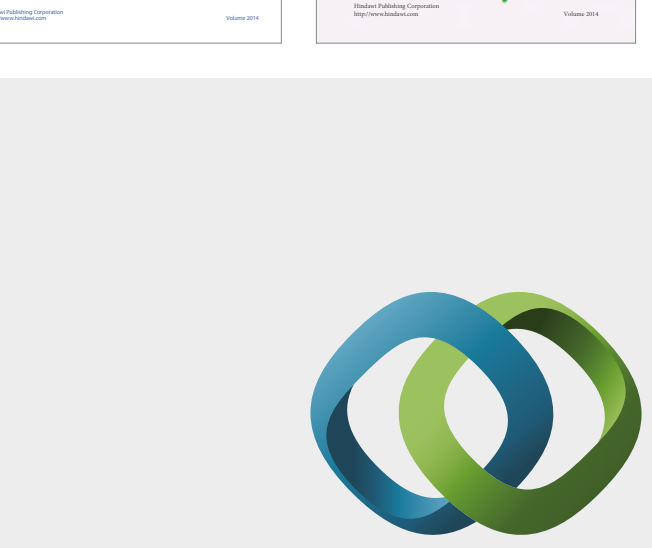

\section{Hindawi}

Submit your manuscripts at

https://www.hindawi.com
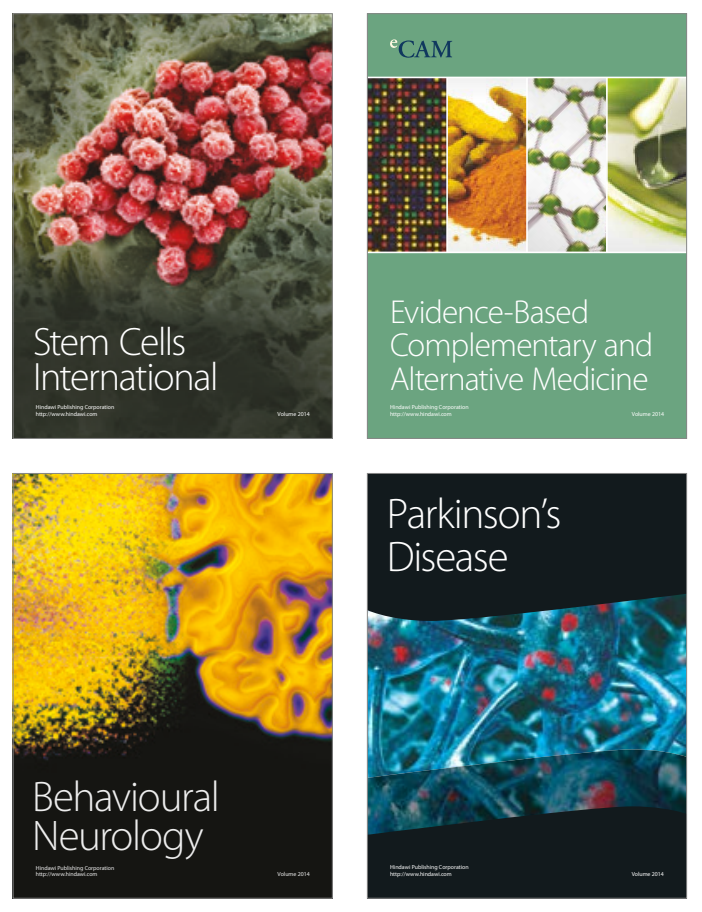
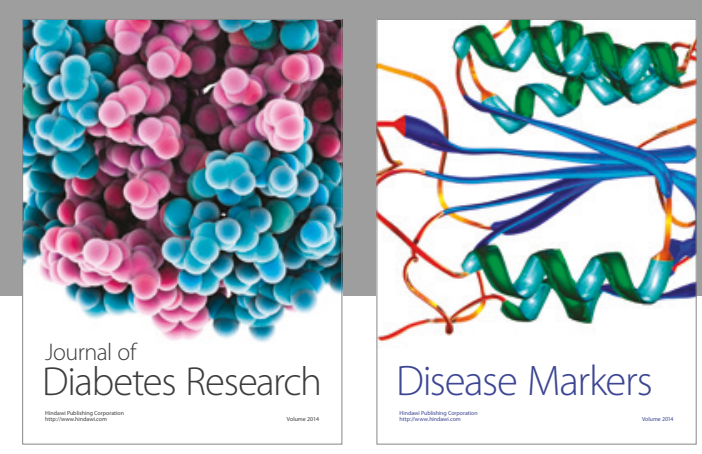

Disease Markers
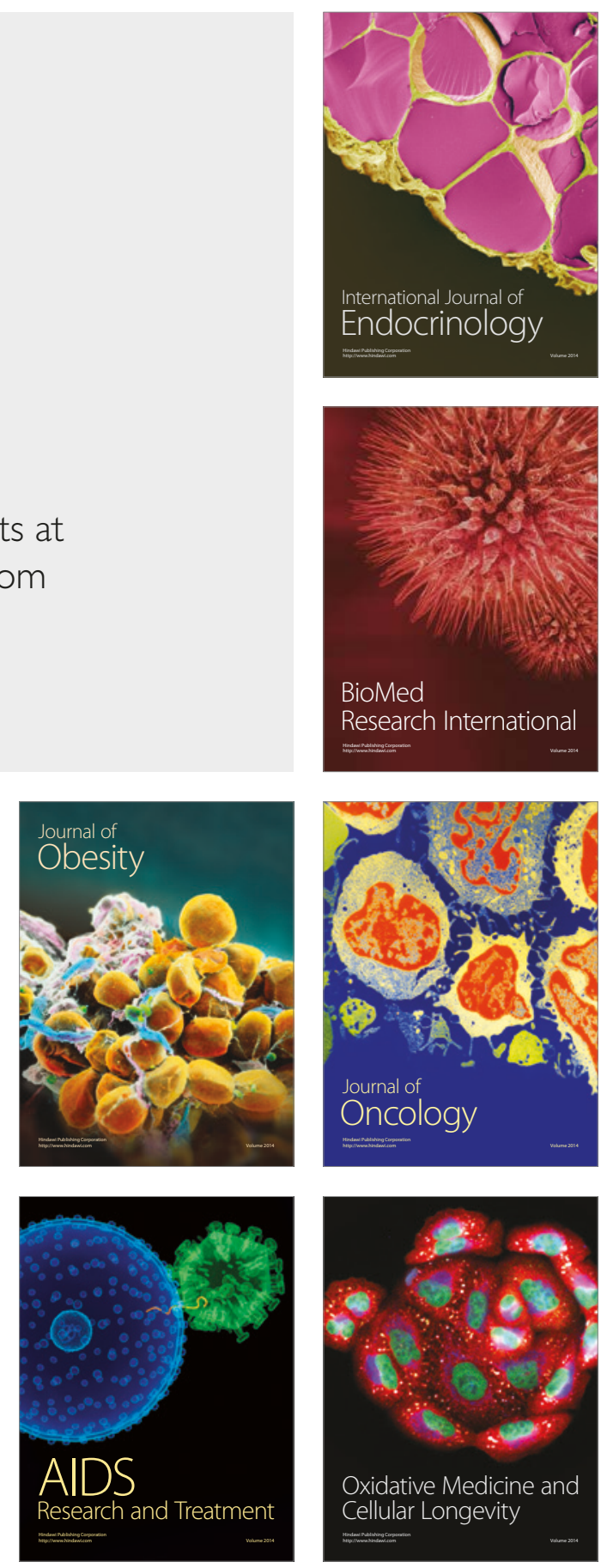